\title{
Robust Frequency and Timing Synchronization for OFDM
}

\author{
Timothy M. Schmidl and Donald C. Cox, Fellow, IEEE
}

\begin{abstract}
A rapid synchronization method is presented for an orthogonal frequency-division multiplexing (OFDM) system using either a continuous transmission or a burst operation over a frequency-selective channel. The presence of a signal can be detected upon the receipt of just one training sequence of two symbols. The start of the frame and the beginning of the symbol can be found, and carrier frequency offsets of many subchannels spacings can be corrected. The algorithms operate near the Cramér-Rao lower bound for the variance of the frequency offset estimate, and the inherent averaging over many subcarriers allows acquisition at very low signal-to-noise ratios (SNR's).
\end{abstract}

Index Terms - Carrier frequency, orthogonal frequencydivision multiplexing, symbol timing estimation.

\section{INTRODUCTION}

$\mathbf{I}$ $\mathrm{N}$ AN orthogonal frequency-division multiplexing (OFDM) system, synchronization at the receiver is one important step that must be performed. This paper describes a method to acquire synchronization for either a continuous stream of data as in a broadcast application or for bursty data as in a wireless local area network (WLAN). In both cases the receiver must continuously scan for incoming data, and rapid acquisition is needed. The ratio of the number of overhead bits for synchronization to the number of message bits must be kept to a minimum, and low-complexity algorithms are needed.

Synchronization of an OFDM signal requires finding the symbol timing and carrier frequency offset. Symbol timing for an OFDM signal is significantly different than for a single carrier signal since there is not an "eye opening" where a best sampling time can be found. Rather there are hundreds or thousands of samples per OFDM symbol since the number of samples necessary is proportional to the number of subcarriers. Finding the symbol timing for OFDM means finding an estimate of where the symbol starts. There is usually some tolerance for symbol timing errors when a cyclic prefix is used to extend the symbol. Synchronization of the carrier frequency at the receiver must be performed very accurately, or there will be loss of orthogonality between the subsymbols. OFDM systems are very sensitive to carrier frequency offsets since they can only tolerate offsets which are a fraction of the

Paper approved by M. Luise, the Editor for Synchronization of the IEEE Communications Society. Manuscript received April 16, 1996; revised February 11, 1997. This work was supported in part by a National Science Foundation Graduate Fellowship. This work was presented in part at the IEEE International Conference on Communications (ICC), Dallas, TX, June 1996.

T. M. Schmidl is with DSP Research and Development Center at Texas Instruments Incorporated, Dallas, TX 75243 USA (e-mail: schmidl@ti.com).

D. C. Cox is with the STAR Laboratory, Department of Electrical Engineering, Stanford University, Stanford, CA 94305-4055 USA (e-mail: dcox@nova.stanford.edu).

Publisher Item Identifier S 0090-6778(97)09083-1. spacing between the subcarriers without a large degradation in system performance [1].

There have been several papers on the subject of synchronization for OFDM in recent years. Moose gives the maximum likelihood estimator for the carrier frequency offset which is calculated in the frequency domain after taking the FFT [2]. He assumes that the symbol timing is known, so he just has to find the carrier frequency offset. The limit of the acquisition range for the carrier frequency offset is $\pm 1 / 2$ the subcarrier spacing. He also describes how to increase this range by using shorter training symbols to find the carrier frequency offset. For example shortening the training symbols by a factor of two would double the range of carrier frequency acquisition. This approach will work to a point, but the estimates get worse as the symbols get shorter because there are fewer samples over which to average, and the training symbols need to be kept longer than the guard interval so that the channel impulse response does not cause distortion when estimating the frequency offset.

Nogami and Nagashima [4] present algorithms to find the carrier frequency offset and sampling rate offset. They use a null symbol where nothing is transmitted for one symbol period so that the drop in received power can be detected to find the beginning of the frame. The carrier frequency offset is found in the frequency domain after applying a Hanning window and taking the FFT. The null symbol is also used in [11]. This extra overhead of using a null symbol is avoided by using the technique described in this paper. If instead of a continuous transmission mode, a burst mode is used, it would be difficult to use a null symbol since there would be no difference between the null symbol and the idle period between bursts.

Van de Beek [3] describes a method of using a correlation with the cyclic prefix to find the symbol timing. If this method were used to find the symbol timing, while using one of the previous methods to find the carrier frequency offset, there would still be a problem of finding the start of the frame to know where the training symbols are located.

Classen introduces a method which jointly finds both the symbol timing and carrier frequency offset [5]. However, it is very computationally complex because it uses a trial and error method where the carrier frequency is incremented in small steps over the entire acquisition range until the correct carrier frequency is found. It is impractical to do the exhaustive search and go through a large amount of computation at each possible carrier frequency offset.

This paper introduces some modifications of Classen's method which both greatly simplify the computation necessary for synchronization and extend the range for the acquisition 


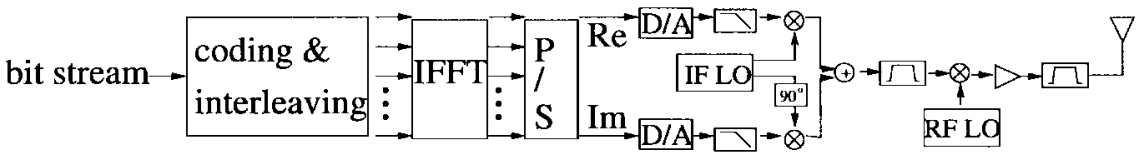

Fig. 1. Block diagram of OFDM transmitter.

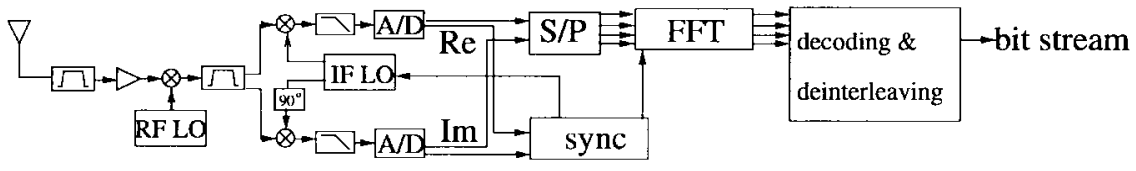

Fig. 2. Block diagram of OFDM receiver.

of carrier frequency offset. The method in this paper avoids the extra overhead of using a null symbol, while allowing a large acquisition range for the carrier frequency offset. By using one unique symbol which has a repetition within half a symbol period, this method can be used for bursts of data to find whether a burst is present and to find the start of the burst.

Acquisition is achieved in two separate steps through the use of a two-symbol training sequence, which will usually be placed at the start of the frame. First the symbol/frame timing is found by searching for a symbol in which the first half is identical to the second half in the time domain. Then the carrier frequency offset is partially corrected, and a correlation with a second symbol is performed to find the carrier frequency offset.

\section{OFDM PRINCIPLES}

The OFDM signal is generated at baseband by taking the inverse fast Fourier transform (IFFT) of quadrature amplitude modulated (QAM) or phase-shift keyed (PSK) subsymbols $c_{k}=a_{k}+j b_{k}$ (Fig. 1). In the figure, the block P/S represents a parallel-to-serial converter. An OFDM symbol has a useful period $T$ and preceding each symbol is a cyclic prefix of length $T_{g}$, which is longer than the channel impulse response so that there will be no intersymbol interference (ISI) [6]. The frequencies of the complex exponentials are $f_{k}=k / T$, and the useful part for $2 N+1$ subcarriers is given by

$$
u(t)=\sum_{k=-N}^{N} c_{k} \exp \left(j 2 \pi f_{k} t\right), \quad 0 \leq t \leq T .
$$

The baseband signal is quadrature modulated, up-converted to the radio frequency (RF) and transmitted through the channel.

At the receiver (Fig. 2), the signal is down-converted to an intermediate frequency (IF), and quadrature demodulated. The block S/P represents a serial-to-parallel converter. A carrier frequency offset of $\Delta f$ causes a phase rotation of $2 \pi t \Delta f$. If uncorrected this causes both a rotation of the constellation and a spread of the constellation points similar to additive white Gaussian noise (AWGN). A symbol-timing error will have little effect as long as all the samples taken are within the length of the cyclically-extended OFDM symbol.

\section{EstimATION OF SyMBOL TIMING}

\section{A. Symbol Timing Estimation Algorithm}

The symbol timing recovery relies on searching for a training symbol with two identical halves in the time do- main, which will remain identical after passing through the channel, except that there will be a phase difference between them caused by the carrier frequency offset. The two halves of the training symbol are made identical (in time order) by transmitting a pseudonoise (PN) sequence on the even frequencies, while zeros are used on the odd frequencies. This means that at each even frequency one of the points of a QPSK constellation is transmitted. In order to maintain an approximately constant signal energy for each symbol the frequency components of this training symbol are multiplied by $\sqrt{2}$ at the transmitter, or the four points of the QPSK constellation are selected from a larger constellation, such as 64-QAM, so that points with higher energy can be used. Transmitted data will not be mistaken as the start of the frame since any actual data must contain odd frequencies. Note that an equivalent method of generating this training symbol is to use an IFFT of half the normal size to generate the time domain samples. The repetition is not generated using the IFFT, so instead of just using the even frequencies, a PN sequence would be transmitted on all of the subcarriers to generate the time domain samples which are half a symbol in duration. These time-domain samples are repeated (and properly scaled) to form the first training symbol.

The second training symbol contains a PN sequence on the odd frequencies to measure these subchannels, and another PN sequence on the even frequencies to help determine frequency offset. Table I illustrates the use of PN sequences in the training sequence for an OFDM signal with nine subcarriers with the points chosen from a subset of a 64QAM constellation. The selection of a particular PN sequence should not have much effect on the performance of the synchronization algorithms. Instead the PN sequence can be chosen on the basis of being easy to implement or having a low peak-to-average power ratio so that there is little distortion in the transmitter amplifier.

Complex samples $r_{m}$ are taken by mixing the received signal down to the IF, splitting the signal into two branches, multiplying by both the in-phase and quadrature local oscillators, and low-pass filtering and sampling to get baseband in-phase and quadrature components (Fig. 2). This can be expressed mathematically by writing the IF local oscillator for the in-phase branch as

$$
v_{i}(t)=2 \cos \left(2 \pi f_{\mathrm{IF}} t\right)
$$


TABLE I

Illustration of Use of PN Sequences for Training Symbols

\begin{tabular}{r|r|r|r}
\hline freq. num. $\mathrm{k}$ & $c_{1, k}$ & $c_{2, k}$ & $v_{k}=\sqrt{2} \frac{c_{2, k}}{c_{1, k}}$ \\
\hline-4 & $7+7 \mathrm{j}$ & $5-5 \mathrm{j}$ & $-\mathrm{j}$ \\
-3 & 0 & $-5-5 \mathrm{j}$ & \\
-2 & $-7+7 \mathrm{j}$ & $-5-5 \mathrm{j}$ & $\mathrm{j}$ \\
-1 & 0 & $-5+5 \mathrm{j}$ & \\
0 & $7+7 \mathrm{j}$ & $-5-5 \mathrm{j}$ & -1 \\
1 & 0 & $5+5 \mathrm{j}$ & \\
2 & $7-7 \mathrm{j}$ & $-5+5 \mathrm{j}$ & -1 \\
3 & 0 & $5-5 \mathrm{j}$ & \\
4 & $7+7 \mathrm{j}$ & $5+5 \mathrm{j}$ & 1 \\
\hline
\end{tabular}

and the IF local oscillator for the quadrature branch at the receiver as

$$
v_{q}(t)=2 \sin \left(2 \pi f_{\mathrm{IF}} t\right)
$$

Let the output of the mixer after down-conversion be $s(t)$. The demodulated signal before the sampler can be expressed as

$$
r(t)=\operatorname{LPF}\left\{s(t) v_{i}(t)\right\}+j \operatorname{LPF}\left\{s(t) v_{q}(t)\right\}
$$

where $\operatorname{LPF}\{\cdot\}$ means to low-pass filter the terms in the argument. The output of the in-phase branch is considered to be real and the output of the quadrature branch is considered to be imaginary. This is a mathematical convention to represent the in-phase and quadrature components as a complex number. After sampling, the complex samples are denoted as $r_{m}$.

Consider the first training symbol where the first half is identical to the second half (in time order), except for a phase shift caused by the carrier frequency offset. If the conjugate of a sample from the first half is multiplied by the corresponding sample from the second half ( $T / 2$ seconds later), the effect of the channel should cancel, and the result will have a phase of approximately $\phi=\pi T \Delta f$. At the start of the frame, the products of each of these pairs of samples will have approximately the same phase, so the magnitude of the sum will be a large value.

Let there be $L$ complex samples in one-half of the first training symbol (excluding the cyclic prefix), and let the sum of the pairs of products be

$$
P(d)=\sum_{m=0}^{L-1}\left(r_{d+m}^{*} r_{d+m+L}\right)
$$

which can be implemented with the iterative formula

$$
P(d+1)=P(d)+\left(r_{d+L}^{*} r_{d+2 L}\right)-\left(r_{d}^{*} r_{d+L}\right) .
$$

Note that $d$ is a time index corresponding to the first sample in a window of $2 L$ samples. This window slides along in time as the receiver searches for the first training symbol. The received energy for the second half-symbol is defined by

$$
R(d)=\sum_{m=0}^{L-1}\left|r_{d+m+L}\right|^{2}
$$

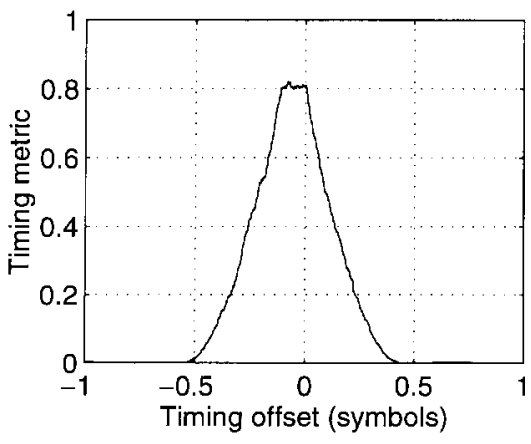

Fig. 3. Example of the timing metric for the AWGN channel $(\mathrm{SNR}=10$ $\mathrm{dB})$.

which can also be calculated iteratively. $R(d)$ may be used as part of an automatic gain control (AGC) loop. A timing metric can be defined as

$$
M(d)=\frac{|P(d)|^{2}}{(R(d))^{2}} .
$$

Fig. 3 shows an example of the timing metric as a window slides past coincidence for the AWGN channel for an OFDM signal with 1000 subcarriers, a carrier frequency offset of 12.4 subcarrier spacings, and an signal-to-noise ratio (SNR) of 10 $\mathrm{dB}$, where the SNR is the total signal (all the subcarriers) to noise power ratio. The timing metric reaches a plateau which has a length equal to the length of the guard interval minus the length of the channel impulse response since there is no ISI within this plateau to distort the signal. For the AWGN channel, there is a window with a length of the guard interval where the metric reaches a maximum, and the start of the frame can be taken to be anywhere within this window without a loss in the received SNR. For the frequency selective channels, the length of the impulse response of the channel is shorter than the guard interval by design choice of the guard interval, so the plateau in the maximum of the timing metric is shorter than for the AWGN channel.

This plateau leads to some uncertainty as to the start of the frame. For the simulations in this paper, OFDM symbols are generated with 1000 frequencies, -500 to 499 . They are slightly oversampled at a rate of 1024 samples for the useful part of each symbol. In an actual hardware implementation, the ratio of the sampling rate to the number of frequencies would be higher to ease filtering requirements. The guard interval is set to about $10 \%$ of the useful part, which is 102 samples.

\section{B. Performance of Symbol Timing Estimator}

There are two issues to consider when evaluating the performance of the symbol timing estimator. First, since the timing metric is also used to determine whether the training sequence has been received, there is a probability of either missing a training sequence and not detecting the signal or falsely detecting a training sequence when none is there. In this paper the distribution of the timing metric at the correct start of the frame is calculated. Using this distribution, the number of samples that need to be processed during the detection phase can be determined and a threshold can be 
set for this detection. Second, there is some degradation in performance if the symbol timing estimate deviates from the correct region. Simulations are performed to find the effect of extra interference that is introduced by poor symbol timing estimates for two types of channels.

1) Distribution of Timing Metric: Let each complex sample $r_{m}=s_{m}+n_{m}$ be made up of a signal and a noise component. Let the variance of the real and imaginary components be:

$$
\begin{aligned}
& E\left[\operatorname{Re}\left\{s_{m}\right\}^{2}\right]=E\left[\operatorname{Im}\left\{s_{m}\right\}^{2}\right]=\sigma_{s}^{2} \\
& E\left[\operatorname{Re}\left\{n_{m}\right\}^{2}\right]=E\left[\operatorname{Im}\left\{n_{m}\right\}^{2}\right]=\sigma_{n}^{2}
\end{aligned}
$$

so that the SNR is $\sigma_{s}^{2} / \sigma_{n}^{2}$. To find the mean and variance of the estimator at the best symbol timing, first look at $P(d)$ which can be written as

$$
\begin{aligned}
P(d)= & \sum_{m=0}^{L-1} s_{d+m}^{*} s_{d+m+L}+s_{d+m}^{*} n_{d+m+L} \\
& +s_{d+m+L} n_{d+m}^{*}+n_{d+m}^{*} n_{d+m+L}
\end{aligned}
$$

At the correct symbol timing, this can be broken into parts that are in-phase and quadrature to the $s_{d_{\mathrm{opt}}+m}^{*} s_{d_{\mathrm{opt}}+m+L}$ product which has phase $\phi$. This is just another way of looking at the problem with a new set of axes with one axis in the $\phi$ direction and the other axis perpendicular to it. For usable values of SNR, when the magnitude is taken the quadrature part will be small compared to the in-phase part and can be neglected, so

$$
\begin{aligned}
\left|P\left(d_{\mathrm{opt}}\right)\right| \approx & e^{-j \phi} \sum_{m=0}^{L-1} s_{d_{\mathrm{opt}}+m}^{*} s_{d_{\mathrm{opt}}+m+L} \\
& +\sum_{m=0}^{L-1} \operatorname{inPhase}_{\phi}\left\{s_{d_{\mathrm{opt}}+m}^{*} n_{d_{\mathrm{opt}}+m+L}\right. \\
& \left.+s_{d_{\mathrm{opt}}+m+L} n_{d_{\mathrm{opt}}+m}^{*}+n_{d_{\mathrm{opt}}+m}^{*} n_{d_{\mathrm{opt}}+m+L}\right\}
\end{aligned}
$$

where inPhase $\operatorname{P}_{\phi}\{\cdot\}$ means the component in the $\phi$ direction. The quadrature part is neglected because the Rician distribution can be approximated by a Gaussian when taking the envelope of a dominant signal with Gaussian noise [8]. The dominant in-phase part is so much larger than the quadrature part that the envelope can be approximated by the in-phase part. Note that the first term of $\left|P\left(d_{\text {opt }}\right)\right|$ is much larger than the second term since the $s_{d_{\mathrm{opt}}+m}^{*} s_{d_{\mathrm{opt}}+m+L}$ products all have phase $\phi$ and add in-phase, while all the other terms add with random phases. By the central limit theorem (CLT), $\left|P\left(d_{\mathrm{opt}}\right)\right|$ is Gaussian with mean $2 L \sigma_{s}^{2}$ since each of the $L$ terms $s_{d_{o p t}+m}^{*} s_{d_{\text {opt }}+m+L}$ has an expected value of $2 \sigma_{s}^{2}$, and all the other terms have an expected value of zero.

For $R\left(d_{\mathrm{opt}}\right)$, the magnitude of each term could be taken by adding the squares of the real and imaginary parts. Instead, we can define a new set of orthogonal axes in which one axis is in the direction of the term $s_{d_{\text {opt }}}+m+L$ and the other is perpendicular to it.

$$
\begin{aligned}
& R\left(d_{\mathrm{opt}}\right) \\
& =\sum_{m=0}^{L-1}\left(\left|s_{d_{\mathrm{opt}}+m+L}\right|+\mathrm{inPhase}_{s_{d_{\mathrm{opt}}+m+L}}\left\{n_{d_{\mathrm{opt}}+m+L}\right\}\right)^{2} \\
& \quad+(\text { quadrature } \\
& \left.\quad\left\{n_{s_{d_{\mathrm{opt}}+m+L}+m+L}\right\}\right)^{2}
\end{aligned}
$$

Here, inPhase ${ }_{s_{d_{\mathrm{opt}}+m+L}}\{\cdot\}$ means to take the component in the direction of $s_{d_{\text {opt }}+m+L}$. By the CLT, $R(d)$ is also Gaussian with mean $2 L\left(\sigma_{s}^{2}+\sigma_{n}^{2}\right)$. Note that for usable values of $\mathrm{SNR}$, the mean is much greater than the standard deviation. Thus, for the Gaussian approximation, the probability of $R\left(d_{\mathrm{opt}}\right)<0$ is insignificantly small making the approximation of the nonnegative $R\left(d_{\mathrm{opt}}\right)$ by a Gaussian reasonable. Another equivalent way of thinking about the distribution is that $R\left(d_{\text {opt }}\right)$ is Rician with the mean much larger than the standard deviation. In this case the Gaussian approximation may be used [8].

Define the square root of $M(d)$ to be $q(d)=|P(d)| / R(d)$ (see (14) at the bottom of the page).

Since the standard deviations of both the numerator and denominator are much smaller than the means, the approximation $a(1+b) /(1+c) \approx a(1+b-c)$ can be used. Another way to explain this is that there is a Gaussian random variable in the numerator and a Gaussian random variable in the denominator, and since the standard deviation of both of these Gaussian random variables is much smaller than their mean values, the above approximation can be used to write their ratio as a single Gaussian random variable. As long as this approximation holds, $q\left(d_{\mathrm{opt}}\right)$ is Gaussian with

$$
\mu_{q}=E\left[q\left(d_{\mathrm{opt}}\right)\right]=\frac{\sigma_{s}^{2}}{\sigma_{s}^{2}+\sigma_{n}^{2}}
$$

This can be justified because linear operations on a Gaussian random variable will result in another Gaussian random variable [9].

When calculating the variance, note that

$$
e^{-j \phi} s_{d_{\mathrm{opt}}+m}^{*} s_{d_{\mathrm{opt}}+m+L}=\left|s_{d_{\mathrm{opt}}+m+L}\right|^{2}
$$

and

$$
\begin{aligned}
& \text { inPhase }_{\phi}\left\{s_{d_{\mathrm{opt}}+m}^{*} n_{d_{\mathrm{opt}}+m+L}\right\} \\
& \quad=\left|s_{d_{\mathrm{opt}}+m+L}\right| \operatorname{inPhase}_{s_{d_{\mathrm{opt}}+m+L}}\left\{n_{d_{\mathrm{opt}}+m+L}\right\} .
\end{aligned}
$$

$$
\begin{aligned}
& q\left(d_{\mathrm{opt}}\right)= \\
& \frac{e^{-j \phi} \sum_{m=0}^{L-1} s_{d_{\mathrm{opt}}+m}^{*} s_{d_{\mathrm{opt}}+m+L}+\sum_{m=0}^{L-1} \operatorname{inPhase}\left\{s_{d_{\mathrm{opt}}+m}^{*} n_{d_{\mathrm{opt}}+m+L}+s_{d_{\mathrm{opt}}+m+L} n_{d_{\mathrm{opt}}+m}^{*}+n_{d_{\mathrm{opt}}+m}^{*} n_{d_{\mathrm{opt}}+m+L}\right\}}{\sum_{m=0}^{L-1}\left(\left|s_{d_{\mathrm{opt}}+m+L}\right|+\text { inPhase }_{s_{d_{\mathrm{opt}}+m+L}}\left\{n_{d_{\mathrm{opt}}+m+L}\right\}\right)^{2}+\left(\text { quadrature }_{s_{d_{\mathrm{opt}}+m+L}}\left\{n_{d_{\mathrm{opt}}+m+L}\right\}\right)^{2}}
\end{aligned}
$$


Since these terms are the same in both the numerator and denominator, they do not contribute to the overall variance. Then

$$
\begin{aligned}
\sigma_{q}^{2}=\operatorname{var}\left[q\left(d_{\mathrm{opt}}\right)\right] & =\frac{2 \sigma_{s}^{2} \sigma_{n}^{2}+2 \sigma_{n}^{4}+\mu_{q}^{2} 2 \sigma_{s}^{2} \sigma_{n}^{2}+\mu_{q}^{2} 4 \sigma_{n}^{4}}{L\left(2 \sigma_{s}^{2}+2 \sigma_{n}^{2}\right)^{2}} \\
& =\frac{\left(1+\mu_{q}^{2}\right) \sigma_{s}^{2} \sigma_{n}^{2}+\left(1+2 \mu_{q}^{2}\right) \sigma_{n}^{4}}{2 L\left(\sigma_{s}^{2}+\sigma_{n}^{2}\right)^{2}}
\end{aligned}
$$

$M\left(d_{\mathrm{opt}}\right)$ is $\left(q\left(d_{\mathrm{opt}}\right)\right)^{2}$, which is the square of a Gaussian random variable. Since the variance is much smaller than the mean, this also can be approximated by a Gaussian random variable since linear operations performed on a Gaussian random variable will produce another Gaussian random variable:

$$
\begin{aligned}
M\left(d_{\mathrm{opt}}\right) & =\left(\mu_{q}+n\left(0, \sigma_{q}^{2}\right)\right)^{2} \\
& =\mu_{q}^{2}+2 \mu_{q} n\left(0, \sigma_{q}^{2}\right)+\left(n\left(0, \sigma_{q}^{2}\right)\right)^{2} \\
& \approx \mu_{q}^{2}+2 \mu_{q} n\left(0, \sigma_{q}^{2}\right)
\end{aligned}
$$

where $n\left(\mu, \sigma^{2}\right)$ is used to denote a Gaussian random variable with a mean of $\mu$ and a variance of $\sigma^{2}$.

The expected value is

$$
\mu_{M}=E\left[M\left(d_{\mathrm{opt}}\right)\right]=\frac{\sigma_{s}^{4}}{\left(\sigma_{s}^{2}+\sigma_{n}^{2}\right)^{2}}
$$

and the variance is

$$
\begin{aligned}
\operatorname{var}\left[M\left(d_{\mathrm{opt}}\right)\right] & =4 \mu_{q}^{2} \sigma_{q}^{2} \\
& =\frac{2 \sigma_{s}^{4}\left[\left(1+\mu_{M}\right) \sigma_{s}^{2} \sigma_{n}^{2}+\left(1+2 \mu_{M}\right) \sigma_{n}^{4}\right]}{L\left(\sigma_{s}^{2}+\sigma_{n}^{2}\right)^{4}} .
\end{aligned}
$$

At high SNR the mean is approximately 1 and the variance is approximately $2 \sigma_{s}^{4}\left[(1+1) \sigma_{s}^{2} \sigma_{n}^{2}\right] /\left[L\left(\sigma_{s}^{2}\right)^{4}\right] \approx 4 /(L \cdot \mathrm{SNR})$.

The value of $M\left(d_{\mathrm{opt}}\right)$ also can give an estimate of the SNR, which is

$$
\widehat{\mathrm{SNR}}=\frac{\sqrt{M\left(d_{\mathrm{opt}}\right)}}{1-\sqrt{M\left(d_{\mathrm{opt}}\right)}}
$$

This equation is derived from (19). This can be written as $\widehat{\mathrm{SNR}}=-1+1 /\left(1-q\left(d_{\mathrm{opt}}\right)\right)$. The denominator $\left(1-q\left(d_{\mathrm{opt}}\right)\right)$ is Gaussian, and its reciprocal will also be Gaussian since the standard deviation is much smaller than the mean for low values of SNR. Again, this is a linear operation on a Gaussian random variable (after the approximation). Then

$$
\begin{aligned}
E[\widehat{\mathrm{SNR}}] & =\frac{\sigma_{s}^{2}}{\sigma_{n}^{2}}=\mathrm{SNR} \\
\operatorname{var}[\widehat{\mathrm{SNR}}] & =\frac{\sigma_{q}^{2}}{\left(1-\mu_{q}\right)^{4}} .
\end{aligned}
$$

This estimator works well for the SNR below $20 \mathrm{~dB}$. Above this level, $M\left(d_{\mathrm{opt}}\right)$ is so close to 1 that an accurate estimate of the SNR can not be determined, but only that the SNR is high. For example, if $M\left(d_{\mathrm{opt}}\right)=0.9$, then $\widehat{\mathrm{SNR}}=18.5=12.7 \mathrm{~dB}$. This can be used to set a threshold so that very weak signals will not be decoded, or it can be used in a WLAN to feed back to the transmitter to indicate what data rate will be supported so that an appropriate constellation and code can be chosen. A lookup table can be implemented based on $M\left(d_{\mathrm{opt}}\right)$, so that no square roots or divisions need to be performed.

Even if there is a frequency selective channel, all the signal energy will go into the signal component term except when the length of the channel impulse response becomes so large that it is longer than the cyclic prefix. At this point, the energy located at longer delays becomes interference and would be added to the noise terms.

At a position outside the first training symbol, the terms in the sum $P\left(d_{\text {outside }}\right)$ add with random phases since there is not a periodicity for samples spaced by $L$ samples. For the purpose of computing the mean and variance of the timing metric, the samples can be considered to be composed of just noise terms since noise terms will also add with random phases, so the statistics will be independent of the SNR. This assumption is verified by the simulation results shown in Fig. 6 which will be explained later in this section. The real and imaginary parts of $P\left(d_{\text {outside }}\right)$ are Gaussian by the CLT. The sum of the square of two zero-mean Gaussian random variables, each with a variance of 1 is a chi-square random variable with two degrees of freedom and is represented by the symbol $\chi_{2}^{2}$. The mean of $\chi_{2}^{2}$ is 2 and the variance is 4 [9]. To simplify the computations, let the variance of the real and imaginary components of $r_{m}$ be:

$$
E\left[\operatorname{Re}\left\{r_{m}\right\}^{2}\right]=E\left[\operatorname{Im}\left\{r_{m}\right\}^{2}\right]=\sigma_{r}^{2} .
$$

Both $\operatorname{Re}\left\{P\left(d_{\text {outside }}\right)\right\}$ and $\operatorname{Im}\left\{P\left(d_{\text {outside }}\right)\right\}$ are Gaussiandistributed with zero mean and a variance of $2 L \sigma_{r}^{4}$. Incorporating this scaling factor, we have

$$
\left|P\left(d_{\text {outside }}\right)\right|^{2}=2 L \sigma_{r}^{4} \chi_{2}^{2}
$$

The mean and variance of $\left|P\left(d_{\text {outside }}\right)\right|^{2}$ are

$$
\begin{aligned}
E\left[\left|P\left(d_{\text {outside }}\right)\right|^{2}\right] & =4 L \sigma_{r}^{4} \\
\operatorname{var}\left[\left|P\left(d_{\text {outside }}\right)\right|^{2}\right] & =16 L^{2} \sigma_{r}^{8} .
\end{aligned}
$$

The denominator $R\left(d_{\text {outside }}\right)$ has a Gaussian distribution by the CLT, and its square is also Gaussian because the standard deviation is much smaller than the mean. Again, this is a linear operation on a Gaussian random variable (using the approximation), so the result is also Gaussian. Thus,

$$
R\left(d_{\text {outside }}\right)^{2} \sim n\left(L^{2}\left(2 \sigma_{r}^{2}\right)^{2}, L^{4}\left(2 \sigma_{r}^{2}\right)^{4}\right)
$$

where the $\sim$ operator means "is distributed." The ratio of these two random variables (after dividing both the numerator and denominator by a constant) is

$$
\begin{aligned}
M\left(d_{\text {outside }}\right) & \sim \frac{\frac{1}{2 L} \chi_{2}^{2}}{n\left(1, \frac{4}{L}\right)} \\
& \approx \frac{1}{2 L} \chi_{2}^{2}-n\left(0, \frac{4}{L^{3}}\right) \\
& \approx \frac{1}{2 L} \chi_{2}^{2}
\end{aligned}
$$

Here, the variance of the Gaussian random variable is proportional to $1 / L^{3}$ and can be neglected. The mean and variance 


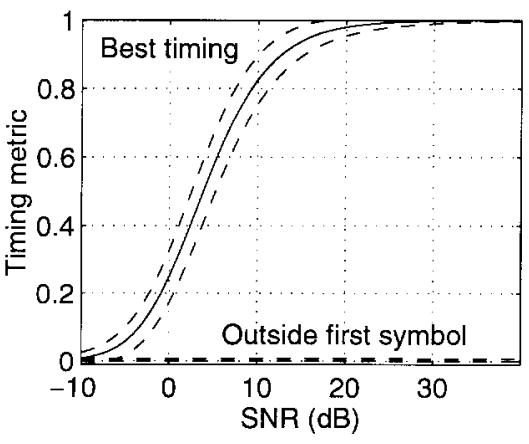

Fig. 4. Expected value of timing metric with $L=512$. Dashed lines indicate three standard deviations.
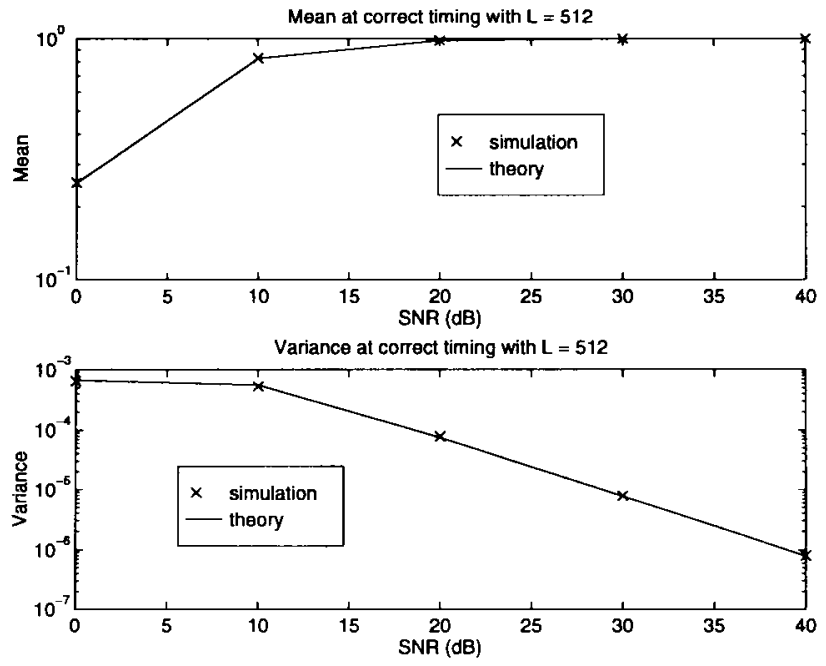

Fig. 5. Mean and variance at the correct timing point with $L=512$ for the exponential channel.

of the timing metric are

$$
\begin{aligned}
E\left[M\left(d_{\text {outside }}\right)\right] & =\frac{1}{L} \\
\operatorname{var}\left[M\left(d_{\text {outside }}\right)\right] & =\frac{1}{L^{2}}
\end{aligned}
$$

Fig. 4 shows a plot of the expected value of timing metric $M(d)$ versus SNR at both the best timing instant and a point outside the training symbol. The dashed lines indicate three standard deviations from each curve.

Figs. 5 and 6 show the results of simulations performed on the exponential frequency selective channels with 1000 subcarriers for the correct timing and an incorrect timing, respectively. The correct timing point was chosen at the start of the useful part of the first training symbol, and the incorrect timing point was chosen one symbol after that. For each SNR value, the simulation was run 10000 times, each time generating different $\mathrm{PN}$ sequences, channels, and noise. Note that the simulated variance is slightly higher than that calculated by theory for the incorrect timing. This is caused because there is a correlation between the signal terms $r_{d+m}^{*}$ and $r_{d+m+L}$, while the calculation assumes that they are independent.

2) Probability of Missing the Training Sequence or of False Detection: The probability distributions calculated in Section
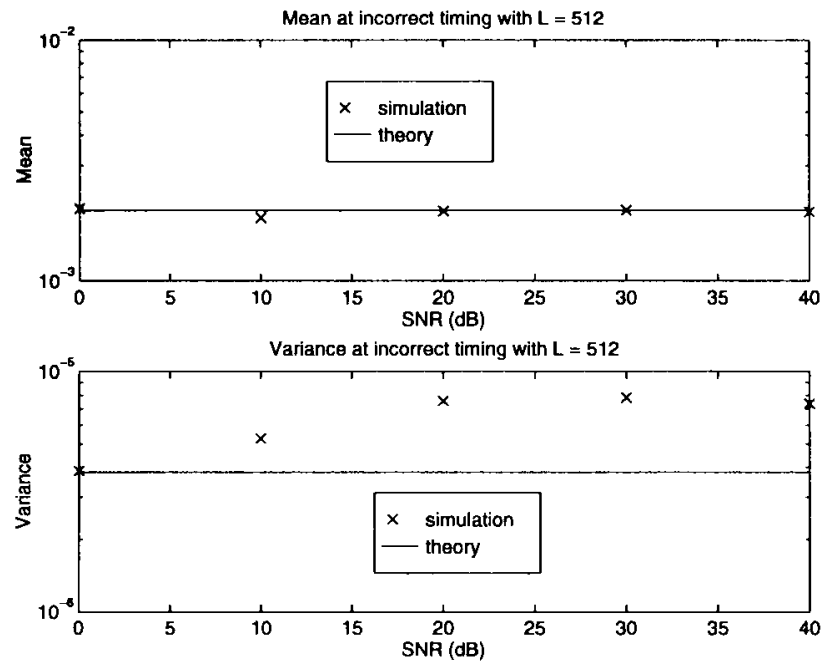

Fig. 6. Mean and variance at an incorrect timing point with $L=512$ for the exponential channel.

III-B-1 can be used to determine both the probability of not detecting a training sequence when one is present and of falsely detecting a training sequence when one is not present. As an example of how this can be done, let there be 1000 subchannels and let $L=512$. If the system is designed to detect a signal if the SNR is at least $10 \mathrm{~dB}$, then from Eqs. (19) and (20), when the signal is present the mean is 0.827 and the variance is $5.58 \times 10^{-4}$, so the standard deviation is $2.36 \times 10^{-2}$ at the correct timing. If no signal is present, then from (28) the mean is $1.95 \times 10^{-3}$ and the standard deviation is also $1.95 \times 10^{-3}$. If the threshold is set at 0.1 , then the margin for error when the training signal is present is

$$
\frac{0.827-0.1}{2.36 \times 10^{-2}}=30 \quad \text { standard deviations. }
$$

Similarly, when the signal is not present, the margin for error is

$$
\frac{0.1-1.95 \times 10^{-3}}{1.95 \times 10^{-3}}=50 \quad \text { standard deviations. }
$$

If the desired probability of error of missing a training sequence is $1 \times 10^{-3}$, this requires that the threshold be 3.1 standard deviations below the mean found with (19). When computing the probability of false detection, note that the training signal is not present for most of each frame. If there are 100 OFDM symbols within one frame, then most of the time the training symbol is not present. Since the sliding windows for the symbol timing estimator are half a symbol long, there can be about 200 uncorrelated values of the symbol timing estimator within one frame. If the probability of false detection within one frame is desired to be $1 \times 10^{-3}$, then the probability of false detection at any point in time should be about $5 \times 10^{-6}$. From [10] the cumulative distribution function for $\chi_{2}^{2}$ is

$$
F\left(\chi_{2}^{2}\right)=1-\exp \left[-\chi_{2}^{2} / 2\right] .
$$

To get a probability of error of $5 \times 10^{-6}$ requires $\chi_{2}^{2}$ to be less than 24.4, which corresponds to

$$
(24.4-2) / 2=11.2 \quad \text { standard deviations. }
$$



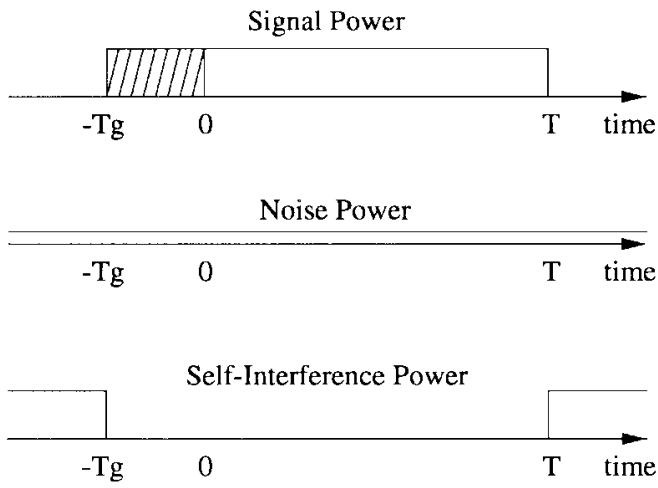

Fig. 7. Relationship of signal, noise, and interference power to the symbol timing position for the AWGN channel. The shaded portion in the first plot indicates the range where the synchronizer can estimate the start of the symbol to maximize SNIR.

In this example, both the probabilities of missing a training sequence or of false detection within one frame are less than $1 \times 10^{-3}$. This allows the threshold to be adjusted to operate at a lower SNR if that is desired. Another option is to reduce the amount of computation performed while searching for the training sequence by not processing every sample. This could be useful in a burst mode when data may not arrive very often.

3) Reduction in SNIR: Two channels are used in simulations to measure the performance of the estimation algorithms. First the AWGN channel is used to show how the algorithms perform on a benign channel, and it is also used as a basis for comparison. Then a frequency selective channel with an exponential power delay profile is used to show that the algorithms perform well in a more realistic environment. Sixteen paths are chosen with path delays of $0,4,8, \cdots, 60$ samples, so the impulse response of the channel is shorter than the guard interval. The amplitude of each path is calculated from the exponential distribution, so

$$
A_{i}=\exp \left(-\tau_{i} / 60\right)
$$

where $A_{i}$ is the amplitude of the $i$ th path and $\tau_{i}$ is the delay of the $i$ th path is samples. The phase of each path is chosen from a uniform distribution from 0 to $2 \pi$.

The reduction in the signal-to-noise-plus-interference ratio (SNIR) due to a symbol timing error can be calculated for the simulated channels. SNIR can be defined as

$$
\mathrm{SNIR}=\frac{\sigma_{s}^{2}}{\sigma_{n}^{2}+\sigma_{i}^{2}}
$$

where $\sigma_{i}^{2}$ is the variance of the intersymbol interference (ISI) added by incorrect symbol timing. Fig. 7 plots the average signal, noise, and interference power levels versus time for an AWGN channel. Note that interference here refers to the ISI from symbols before and after the training symbol in the time domain. The time from $-T_{g}$ to 0 is the length of the guard interval, and the time from 0 to $T$ is the length of the useful part of the OFDM symbol. If the synchronizer estimates that the useful part of the symbol starts at any time within the guard interval, which is the shaded area in the first plot of Fig. 7, then there is no reduction in SNIR due to incorrect symbol timing. However, if the synchronizer estimates that the start
TABLE II

Reduction in SNIR (dB) Due to Errors in Symbol Timing

\begin{tabular}{l|r|r|r|r}
\hline & \multicolumn{2}{|c|}{ AWGN } & \multicolumn{2}{c}{ Exponential } \\
\hline SNR (dB) & peak & avg 90\% & peak & avg 90\% \\
\hline 0 & 0.0087 & 0.0007 & 0.0213 & 0.0034 \\
10 & 0.0060 & 0.0000 & 0.0336 & 0.0003 \\
20 & 0.0364 & 0.0000 & 0.1828 & 0.0014 \\
30 & 0.3068 & 0.0000 & 1.2374 & 0.0119 \\
40 & 0.9023 & 0.0000 & 4.1953 & 0.0599 \\
\hline
\end{tabular}

of the symbol is outside the guard interval, there will be both a decrease in signal energy and an increase in interference, resulting in a lower SNIR. This occurs because samples from the previous or next symbol are input into the FFT along with samples from the current symbol. For example, if the start of the symbol is estimated to be at either time $T / 11$ or at time $-T_{g}-T / 11$, then the signal-to-interference ratio (SIR) is 10 , assuming that the interference level is the same as the signal level, as would be the case with a continuous transmission but not with bursts of data. The SNIR will be lower than 10 when the noise is included. For the simulations in this paper, it is assumed that a continuous transmission format is used because this represents the worst case in terms of selfinterference. With multipath delay spread, the symbol timing tolerance window from $-T_{g}$ to 0 is reduced by the length of the channel impulse response.

Two methods to determine the symbol timing are compared on the basis of reduction in SNIR. The first method is to simply find the maximum of the timing metric. The second method is to find the maximum, find the points to the left and right in the time domain, which are $90 \%$ of the maximum, and average these two $90 \%$ times to find the symbol timing estimate. The rationale behind this method is that the best timing points typically lie in a plateau. By trying to determine the center of this plateau, it is more likely that the estimate will not fall slightly off the plateau. Table II compares the two methods on the basis of reduction in SNIR for the AWGN and frequency selective channel. For each type of channel, 10000 simulations are run at each SNR, and in each run, different PN sequences, channels, and noise are generated. The averaging method performs significantly better than simply finding the peak of the timing metric, and it involves only slightly more computation. With a 40-dB SNR, a symbol timing offset of one sample away from the plateau would result in about $10 \mathrm{~dB}$ in degradation in SNIR for the AWGN channel. The averaging method of finding the symbol time resulted in no degradation in 10000 runs for the AWGN channel and a degradation of just under $0.06 \mathrm{~dB}$ for the exponential channel at an SNR of $40 \mathrm{~dB}$.

\section{Estimation OF CARRIER FREQUenCy OfFSET}

\section{A. Carrier Frequency Offset Estimation Algorithm}

The main difference between the two halves of the first training symbol will be a phase difference of

$$
\phi=\pi T \Delta f
$$

which can be estimated by

$$
\hat{\phi}=\operatorname{angle}(P(d))
$$


near the best timing point. If $|\hat{\phi}|$ can be guaranteed to be less than $\pi$, then the frequency offset estimate is

$$
\widehat{\Delta f}=\hat{\phi} /(\pi T)
$$

and the even PN frequencies on the second training symbol would not be needed. Otherwise, the actual frequency offset would be

$$
\frac{\phi}{\pi T}+\frac{2 z}{T}
$$

where $z$ is an integer. By partially correcting the frequency offset, adjacent carrier interference (ACI) can be avoided, and then the remaining offset of $2 z / T$ can be found. After the two training symbols are frequency corrected by $\hat{\phi} /(\pi T)$ (by multiplying the samples by $\exp (-j 2 t \hat{\phi} / T)$ ), let their FFT's be $x_{1, k}$ and $x_{2, k}$, and let the differentially-modulated PN sequence on the even frequencies of the second training symbol be $v_{k}$ (as illustrated in Table I). The PN sequence $v_{k}$ will appear at the output except it will be shifted by $2 z$ positions because of the uncompensated frequency shift of $2 z / T$. Note that because there is a guard interval and there is still a frequency offset, even if there were no differential modulation between training symbols 1 and 2 (i.e., PN sequence $v_{k} \equiv 1$ ), there would still be a phase shift between $x_{1, k}$ and $x_{2, k}$ of $2 \pi\left(T+T_{g}\right) 2 z / T$. Since at this point the integer $z$ is unknown, this additional phase shift is unknown. However, since the phase shift is the same for each pair of frequencies, a metric similar to (8) can be used. Let $X$ be the set of indices for the even frequency components, $X=\{-W,-W+2, \cdots,-4,-2,2,4, \cdots, W-2, W\}$. The number of even positions shifted can be calculated by finding $\hat{g}$ to maximize

$$
B(g)=\frac{\left|\sum_{k \in X} x_{1, k+2 g}^{*} v_{k}^{*} x_{2, k+2 g}\right|^{2}}{2\left(\sum_{k \in X}\left|x_{2, k}\right|^{2}\right)^{2}}
$$

with integer $g$ spanning the range of possible frequency offsets and $W$ being the number of even frequencies with the PN sequence. Then the frequency offset estimate would be

$$
\widehat{\Delta f}=[\hat{\phi} /(\pi T)]+(2 \hat{g} / T) \text {. }
$$

\section{B. Performance of Carrier Frequency Offset Estimator}

Since the carrier frequency offset is found in two steps, by finding the fractional and then integer part of the offset, there are two issues to consider in evaluating the carrier frequency offset estimator. First the variance of the estimator of the fractional part of the frequency offset can be found. Second, the distribution of the carrier offset metric can be found for both the correct and incorrect integer offsets to determine the probability of making an error in finding the integer part of the carrier frequency offset.

1) Variance of Fractional Part of Carrier Frequency Offset Estimator: Using the method in [2]

$$
\operatorname{var}[\hat{\phi} / \pi]=\frac{1}{\pi^{2} \cdot L \cdot \mathrm{SNR}} .
$$

Since the carrier frequency offset estimate (in subcarrier spacings) is made up of the sum of the initial estimate and an even integer, the variance of the initial estimate, $\hat{\phi} / \pi$, will be equal to the variance of the final estimate if the integer $\hat{g}$ is

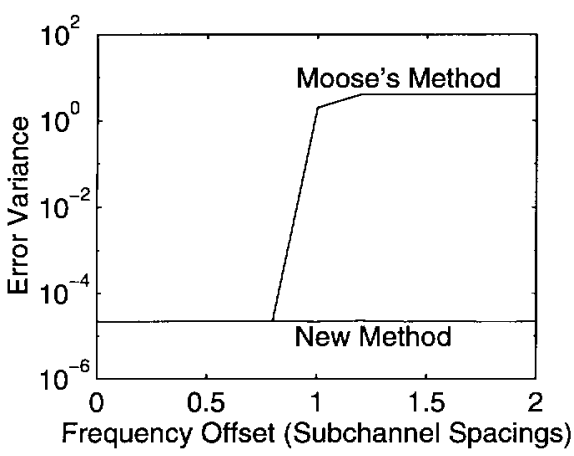

Fig. 8. Comparison of Moose's method and new method of estimating frequency offset.

equal to $g_{\text {correct. }}$. The effect of the remaining frequency offset on the system performance is discussed in [2] and will not be discussed here. By using the method in [7], the Cramér-Rao bound is found to be

$$
\operatorname{var}[\hat{\phi} / \pi] \geq \frac{1}{\pi^{2} \cdot L \cdot \mathrm{SNR}} .
$$

This is not surprising because Moose shows that his estimator is the maximum-likelihood estimator (MLE) of differential phase, and Rife states that the Cramér-Rao bounds are almost met by the MLE with high SNR. Since the frequency offset estimate is made by averaging over hundreds or thousands of subcarriers, the effective SNR is usually very high. To illustrate that the frequency acquisition range is greatly widened with this new method, Fig. 8 compares the error variance for Moose's frequency estimation methods (with two repeated half-symbols) with the new method for 1000 carriers with an SNR of $10 \mathrm{~dB}$. The simulations were performed with 10000 runs per frequency offset value. Since Moose's method is designed to work only with a very small frequency offset, it fails for larger frequency offsets. In Fig. 8, the Cramér-Rao bound is $2.03 \times 10^{-5}$. Note that the simulation here for Moose's algorithm uses the shortened symbols as described in [2], which results is a frequency acquisition range of \pm 1 subchannel spacing.

Fig. 9 compares the error variance for the carrier frequency offset estimate to the Cramér-Rao bound for different values of SNR. In the simulation the carrier frequency offset was set to 2.4 subcarrier spacings so the estimator has to get the integer part correct and get a good estimate of the fractional part of the carrier frequency offset to make a good overall estimate. Again, 10000 trials were run at each SNR value.

2) Distribution of Frequency Offset Metric: The expected values and variances of the estimator $B(g)$ can be calculated in a similar method as in Section III-B-2 to determine if $g_{\text {correct }}$ can be found reliably. At the correct frequency offset, all the signal products $s_{1, h+g_{\text {corroct }}}^{*} v_{h}^{*} s_{2, h+g_{\text {corroct }}}$ have the same phase and

$$
\mu_{B}=E\left[B\left(g_{\text {correct }}\right)\right]=\frac{\sigma_{s}^{4}}{\left(\sigma_{s}^{2}+\sigma_{n}^{2}\right)^{2}}
$$

$$
\operatorname{var}\left[B\left(g_{\text {correct }}\right)\right]=\frac{\sigma_{s}^{4}\left[\left(2+2 \mu_{B}\right) \sigma_{s}^{2} \sigma_{n}^{2}+\left(1+4 \mu_{B}\right) \sigma_{n}^{4}\right]}{W\left(\sigma_{s}^{2}+\sigma_{n}^{2}\right)^{4}} .
$$




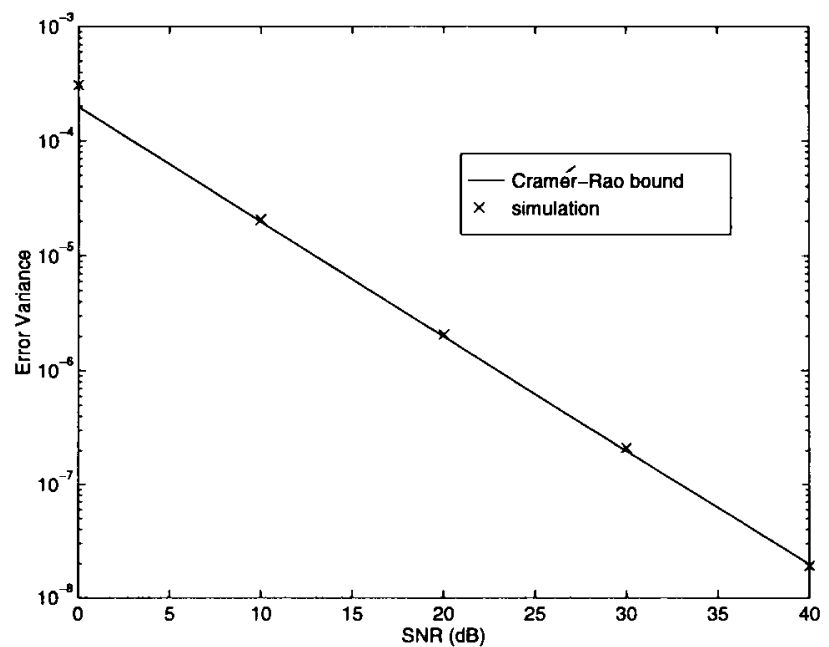

Fig. 9. Comparison of carrier frequency offset estimate to Cramér-Rao bound.

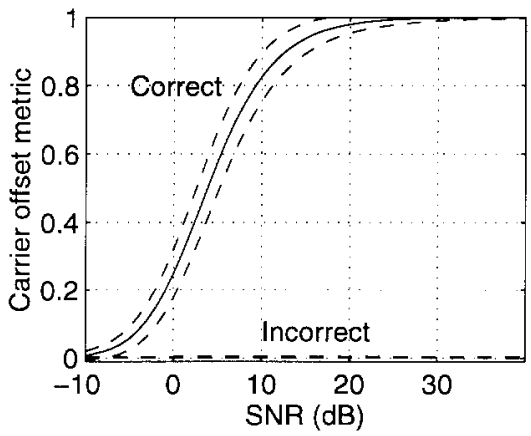

Fig. 10. Expected value of carrier frequency offset metric with $W=500$. Dashed lines indicate three standard deviations.

At high SNR the mean is approximately 1 , and the variance is approximately $\frac{4}{W \cdot \mathrm{SNR}}$.

At an incorrect frequency offset the signal products no longer add in phase, and $B\left(g_{\text {incorrect }}\right)$ has a chi-square distribution with two degrees of freedom with

$$
\begin{aligned}
E\left[B\left(g_{\text {incorrect }}\right)\right] & =\frac{1}{2 W}\left(1+\frac{\sigma_{s}^{2}}{\sigma_{s}^{2}+\sigma_{n}^{2}}\right)<\frac{1}{W} \\
\operatorname{var}\left[B\left(g_{\text {incorrect }}\right)\right] & =\frac{1}{4 W^{2}}\left(1+\frac{3 \sigma_{s}^{4}+2 \sigma_{s}^{2} \sigma_{n}^{2}}{\left(\sigma_{s}^{2}+\sigma_{n}^{2}\right)^{2}}\right)<\frac{1}{W^{2}} .
\end{aligned}
$$

Fig. 10 shows a plot of the expected value of frequency offset metric $B(g)$ versus SNR for both a correct and incorrect frequency offset for the 1000 subcarrier OFDM signal. The dashed lines indicate three standard deviations from each curve.

\section{CONCLUSION}

A method has been presented for the rapid and robust synchronization of OFDM signals, and acquisition is obtained upon the receipt of just one training sequence. By averaging over all the subchannels, it works well in frequency selective channels. This method also gives very accurate estimates of symbol timing and carrier frequency offset and provides a very wide acquisition range for the carrier frequency offset. It also provides an estimate of the SNR, and the probability of false locks or missing the training symbols is very low. For a wireless LAN, such a fast and low-overhead synchronization process is necessary because there will be only one training sequence transmitted in each burst for synchronization.

\section{REFERENCES}

[1] T. Pollet, M. Van Bladel, and M. Moeneclaey, "BER sensitivity of OFDM systems to carrier frequency offset and Weiner phase noise," IEEE Trans. Commun., vol. 43, pt. 1, pp. 191-193, Feb.-Apr. 1995.

[2] P. Moose, "A technique for orthogonal frequency division multiplexing frequency offset correction," IEEE Trans. Commun., vol. 42, pp. 2908-2914, Oct. 1994

[3] J.-J. van de Beek, M. Sandell, M. Isaksson, and P. Börjesson, "Lowcomplex frame synchronization in OFDM systems," in Proc. ICUPC, Nov. 6-10, 1995, pp. 982-986.

[4] H. Nogami and T. Nagashima, "A frequency and timing period acquisition technique for OFDM systems," Personal, Indoor and Mobile Radio Commun. (PIRMC), pp. 1010-1015, Sept. 27-29, 1995.

[5] F. Classen and H. Meyr, "Synchronization algorithms for an OFDM system for mobile communication," ITG-Fachtagung, pp. 105-113, Oct. 26-28, 1994.

[6] B. Le Floch, R. Halbert-Lassalle, and D. Castelain, "Digital sound broadcasting to mobile receivers," IEEE Trans. Consumer Electron., vol. 35, pp. 493-503, Aug. 1989.

[7] D. Rife and R. Boorstyn, "Single-tone parameter estimation from discrete-time observations," IEEE Trans. Inform. Theory, vol. IT-20, pp. 591-598, Sept. 1974.

[8] T. Rappaport, Wireless Communications: Principles and Practice. Englewood Cliffs, NJ: Prentice-Hall, 1996

[9] R. Walpole and R. Myers, Probability and Statistics for Engineers and Scientists. New York: Macmillan, 1985.

[10] W. Beyer, CRC Standard Mathematical Tables. New York: CRC Press, 1983.

[11] P. de Bot, B. Le Floch, V. Mignone, and H.-D. Schütte, "An overview of the modulation and channel coding schemes developed for digital terrestrial television broadcasting within the dTTb project," in Int. Broadcasting Conv., Sept. 16-20, 1994, pp. 569-576.

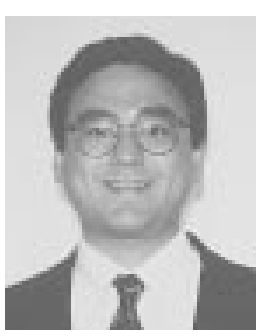

Timothy M. Schmidl received the B.S. degree (with highest honors) in electrical engineering from the University of Texas at Austin in 1992, and the M.S. and Ph.D. degrees in electrical engineering from Stanford University, Stanford, CA, in 1994 and 1997, respectively.

From 1993 to 1997, he was a Research Assistant in the Information Systems Laboratory and the STAR Laboratory at Stanford University. In 1997 he joined the Digital Communication Technology group of the Digital Signal Processing Solutions Research \& Development Center at Texas Instruments and is currently a Member of the Technical Staff.

Donald C. Cox (S'58-M'61-SM'72-F'79), for photograph and biography, see p. 884 of the July 1997 issue of this TransaCtions. 